\title{
Competencia para las relaciones sentimentales en el proceso de cortejo adolescente: un estudio descriptivo
}

\section{Romantic competence during the courtship process: a descriptive study}

Fecha de recepción: 27-05-2021

Fecha de aceptación: 01-12-2021
Carmen Viejo

Universidad de Córdoba.

Noemí Toledano

Universidad de Córdoba

Mercedes Gómez-López

Universidad de Córdoba

Rosario Ortega-Ruiz

Universidad de Córdoba

\section{resumen/alsstract:}

El éxito de las relaciones sentimentales adolescentes estará determinado por el dominio de ciertas habilidades de carácter bidireccional que requieren del desarrollo individual e interpersonal, tales como habilidades para el inicio de la relación, para ser asertivo/a y decir no, para la autorrevelación, para el apoyo emocional y para la gestión de conflictos. Para analizar la percepción que los y las adolescentes tienen sobre sus propias habilidades -denominadas en conjunto como Competencia Sentimental- en el momento de iniciar sus primeras relaciones, 513 adolescentes (49\% chicos) de 13-19 años participaron en este estudio. Los resultados señalaron un nivel medio en la percepción de competencia sentimental, siendo la habilidad para compartir información personal con la persona que te gusta (autorrevelación) la que obtenía las menores puntuaciones, y la habilidad de dar apoyo emocional las puntuaciones más altas. La variable sexo apenas demostró diferencias significativas, siendo que chicos y chicas reportaron sentirse más o menos igual de competentes en sus relaciones sentimentales. La edad indicó ser una variable que incidía en la mayoría de las dimensiones, contribuyendo al desarrollo de la competencia sentimental. Los resultados de este estudio descriptivo subrayan la necesidad de apoyar a los y las jóvenes en su proceso de maduración sentimental y de desarrollo competencial para la formación de relaciones sentimentales ajustadas y de calidad.

Successful adolescent romantic relationships depend on the mastery of certain bidirectional skills that require individual and interpersonal development, such as skills for the beginning of the relationship, to be assertive and say no, for selfdisclosure, for emotional support and conflict management. In order to analyse the perception that adolescents have of their own abilities - understood as Romantic Competence - at the time of initiating their first relationships, during the courtship process, 513 adolescents (49\% boys) aged 13-19 years participated in this study. The results indicated a medium level in the perception of romantic competence, being the ability to share personal information with the person you like (self-disclosure) the one that obtained the lowest scores and the ability to give emotional support the highest. The sex variable barely showed significant differences, where boys and girls reported feeling similarly competent in their romantic relationships. Age indicated to be a variable that affected most of the dimensions, contributing to the development of romantic competence. The results of this descriptive study highlight the need to support young people in their process of romantic maturation and competence development for the formation of high-quality and healthy romantic relationships.

\section{palabras clave/keywords:}

Adolescencia, parejas, calidad de las relaciones, competencia social

Adolescence, dating, relationship quality, social competence 


\section{Introducción}

La formación de las primeras relaciones sentimentales supone un reto en el desarrollo de los y las adolescentes. Se trata de un importante hito psicosocial en cuanto que abre el escenario de un nuevo ecosistema de relaciones que puede saldar con beneficiosos resultados para el desarrollo general de los chicos y chicas, pero que no es ajeno al riesgo de comportamientos desadaptados o violentos (Gómez-López et al., 2019). Algunos de estos comportamientos podrían, incluso, significar la antesala de otros problemas que están siendo visualizados como grandes problemas sociales y de salud, como la violencia de género (Haselschwerdt et al., 2017). Por ello, la necesidad de recursos educativos y sociales que apoyen a los y las jóvenes en la tarea de crecimiento y aprendizaje que supone el proceso de cortejo y la satisfactoria formación de las primeras relaciones de pareja se dibuja como una de las demandas sociales del siglo XXI.

La adolescencia es una etapa del ciclo vital caracterizada por importantes cambios psicosociales que afectan a la percepción global del individuo y a la definición de su propia identidad. En esta etapa, muchos cambios presentan retos de aprendizaje y desarrollo, pero los que se refieren al ajuste social y la calidad de la vida adolescente son prioritarios (Gorrese y Ruggieri, 2012). Comparados con los niños y niñas, los y las adolescentes dan más importancia a las relaciones que se forman dentro del grupo de iguales, siendo estas relaciones más complejas y jerarquizadas, y son más sensibles a la aceptación o rechazo por parte de su grupo de referencia (Blakemore, 2008). Aunque durante estos años la vida familiar presenta retos importantes para la convivencia, es en el grupo de los iguales -particularmente en la vida escolar- en el que encontramos mayores desafíos para el buen desarrollo psicosocial del/la adolescente. Estudios recientes han señalado que procesos evolutivos como el cortejo y el inicio de las primeras relaciones sentimentales, asunto en el que los y las adolescentes se involucran profundamente, podrían incidir en la vida social y en la convivencia de los centros educativos en la medida en que suponen una nueva forma de interacción social para los chicos y chicas (Finkel et al., 2017; Savickait et al., 2020).

La maduración sexual, el aumento de los niveles hormonales y el desarrollo de los caracteres sexuales secundarios, además de dotar al adolescente de un cuerpo adulto, provocarán el aumento y desarrollo de deseos y pulsiones sexuales que son nuevos e inexplorados (Pringle et al., 2017). Estos cambios empujarán a los chicos y chicas a expresar y experimentar una nueva dimensión en sus relaciones interpersonales y sociales, el aspecto erótico-sentimental, materializado en un proceso de seducción, cortejo y acercamiento a otras personas por quienes se siente un interés romántico. Desde una perspectiva psicoevolutiva, este proceso de juego erótico constituye un continuo que va desde las relaciones de amistad con los iguales y atracción e intimidad con algunos compañeros más que con otros, al inicio propiamente del cortejo y el establecimiento de las primeras relaciones de pareja (Connolly et al., 2000; Dunphy, 1963; Savickait et al., 2020).

Aunque tengan comienzos poco estables, en estas relaciones acontecen fenómenos de intimidad, afecto y apoyo mutuo que, unidos al impulso erótico, pronto pasan a ser procesos sentimentales complejos y significativos que impactan y repercuten en la vida de los ado- 
lescentes. Para el éxito de esta tarea psicoevolutiva será necesaria, por tanto, la adquisición de una serie de competencias sociales dirigidas específicamente a este nuevo contexto de relación interpersonal y social. Las primeras experiencias erótico-sentimentales se revelan como una suerte de entramado complejo, pero descifrable por los protagonistas, en el que se ponen en juego competencias sociales adquiridas con anterioridad, tales como la empatía, la asertividad o el apoyo emocional, que, aunque han sido puestas en práctica en el grupo de iguales, facilitan una adaptación o ajuste social del individuo al contexto específico del cortejo (Romera et al., 2016). Todo ello contribuye a conformar la base competencial sobre la cual deberán desarrollarse las nuevas experiencias sentimentales (Delgado et al., 2011; Viejo et al., 2020).

Estas primeras experiencias erótico-sentimentales podrían suponer, en ocasiones, acontecimientos estresantes para los y las adolescentes (Moksnes y Haugan, 2015) que no disponen -o no tienen conciencia de ello- de recursos eficaces para la finalidad que los motiva. Las relaciones erótico-sentimentales, son intrínsecamente desafiantes y, a menudo, requieren habilidades para afrontar circunstancias como el rechazo, el conflicto o la ruptura (Stroud y Davila, 2008; Viejo et al., 2020); habilidades que se encuentran tanto en las características personales como en las que afloran del contexto social inmediato (Bouchey, 2007; Davila et al., 2017).

La inexperiencia de los chicos y chicas en este contexto los situaría ante el riesgo de desarrollar comportamientos rudos o violentos dentro de la dinámica de formación y establecimiento de la pareja (Pellegrini, 2010), así como a enfrentar dificultades tales como la inclusión o normalización de la violencia. Algunos autores han subrayado a este respecto el papel que juega el uso de ciertos comportamientos agresivos de baja intensidad en el proceso de cortejo. Efectivamente, en el contexto de los iguales, generalmente entre los chicos, determinados comportamientos de exhibición de fuerza o prestigio social, como por ejemplo darse empujones a modo de juego, han sido ampliamente utilizados para denotar el deseo de ganar prestigio o popularidad (Pellegrini, 2010). En el inicio de la pubertad y el despertar de los intereses erótico-sentimentales, parece que estos comportamientos asumen una funcionalidad concreta: atraer la atención de la persona con la que se desea iniciar un acercamiento (Foshee et al., 2011). Esta suerte de juego erótico y expresión de interés, connotado de un cierto componente agresivo, ha sido denominado como dirty dating (OrtegaRuiz y Sánchez, 2011), haciendo referencia a un comportamiento que, aunque rudo, podría ser bien aceptado por ambos miembros de la pareja si el interés resulta mutuo, dejando jugar un papel importante a la interpretación que se hace de dicho comportamiento (Pellegrini, 2003; Viejo, 2014).

El inicio y el mantenimiento de relaciones sentimentales de calidad positiva en la adolescencia se ven determinados por la adquisición y puesta en práctica de habilidades con un carácter bidireccional que requieren tanto del desarrollo individual como interpersonal (Davila et al., 2021): el desarrollo de un buen ajuste normativo, de toma de perspectiva, la competencia social, la toma de decisiones adaptativa, unos altos niveles de empatía y asertividad, o una buena gestión de la popularidad en el grupo revertirán en el desarrollo de lo que algunos autores han denominado competencia sentimental-Romantic Competence 
en la literatura internacional (Davila et al., 2009)-. En el marco del proceso de cortejo, una buena gestión de la competencia relacional para la vida íntima (Shulman et al., 2011), dentro del ámbito de la competencia social, partiría del desarrollo de habilidades en dominios como el inicio propiamente de la relación, las habilidades asertivas y para decir no, la autorrevelación o capacidad para contarle a otra persona cosas íntimas o personales, el apoyo emocional y la gestión de conflictos (Viejo et al., 2020; Zhou et al., 2021). Trabajos previos han demostrado que la percepción de autoeficacia juega un papel clave en este proceso, ya que sentirse seguro sobre la capacidad de uno mismo para gestionar una situación incierta y, quizás, muy deseada, y tener un autoconcepto positivo facilita tomar decisiones que, en muchas ocasiones, están vinculadas no solo a cómo comenzar la interacción con otra persona, sino a cómo continuar y gestionar algunas de las inesperadas situaciones que puedan darse (Buhrmester, 1990; Buhrmester et al., 1988). Comportamientos como iniciar una conversación, llamar por teléfono, pedir salir o llevar la contraria a alguien por quien se siente atracción no siempre resulta sencillo; la competencia interpersonal que se despliega en el marco del proceso de cortejo adolescente se evidencia como una competencia multidimensional que requiere de habilidades personales puestas al servicio de relaciones generalmente diádicas que suponen retos sentimentales de alto calado en la identidad propia, o al menos en el autoconcepto, en un periodo del ciclo vital caracterizado por tener muchos frentes a qué atender en el proceso de desarrollo.

\section{El presente estudio}

La literatura científica sobre el complejo proceso de iniciación a las relaciones de pareja en los años de la adolescencia ha dejado establecido que este es un problema central que focaliza muchas de las preocupaciones sociales en estos años (Haselschwerdt et al., 2017; Finkel et al., 2017; Savickaite et al., 2020). A su vez, la mayoría de los estudios realizados hasta el momento (Gorrese y Ruggieri, 2012; Blakemore, 2008; Viejo et al., 2020) están indicando que la competencia para asumir los sentimientos propios y canalizarlos adecuadamente hacia la gestión positiva de la relación se convierte en un factor de desarrollo y aprendizaje que resulta relevante para el bienestar y el progreso evolutivo de los adolescentes. El proceso de canalización de los cambios físicos, psicológicos y sociales que tienen lugar en los años adolescentes, hacia las relaciones interpersonales y más concretamente hacia el cortejo, presenta dificultades que se abordan mejor cuando el/la adolescente sabe encontrar en sí mismo/a herramientas de comunicación y de afianzamiento de la relación de forma que le resulte positiva y gratificante. Pero la inexperiencia de los y las protagonistas puede ser un factor que diferencie, a unos y otras, en el uso de estos recursos comunicativos y sociales

El objetivo del presente estudio avanza en esta línea de trabajos, buscando analizar la percepción del dominio competencial que los chicos y chicas tienen ante las situaciones de cortejo. Para ello, se desarrolla un estudio descriptivo que pretende dar cuenta del dominio percibido en cada una de las dimensiones consideradas parte de la competencia interpersonal para el cortejo: iniciar relaciones, asertividad y capacidad para decir no, autorrevelación, dar apoyo emocional y gestionar conflictos. En todos los casos, se busca matizar las posi- 
bles diferencias que el sexo y edad de los y las jóvenes pueden estar señalando. Los trabajos revisados, aun sin ser propios del contexto de cortejo, permiten hipotetizar que los chicos y chicas adolescentes serán optimistas en cuanto a su competencia sentimental y presentarán buenos índices en todas las categorías; las chicas, por su parte, serán quienes presenten puntuaciones más altas en las dimensiones que implican gestión emocional o de situaciones conflictivas, mientras que los chicos serán quienes puntúen más alto en las dimensiones más instrumentales para el inicio del contacto, como el inicio propiamente de la relación.

\section{Metodología}

\section{Participantes}

En el estudio participaron 513 adolescentes ( $49 \%$ chicos; $51 \%$ chicas) de edades comprendidas entre los 13 y 19 años $(M=14.86 ; D T=1.09)$, escolarizados en 6 centros públicos de Córdoba (Andalucía) en Educación Secundaria Obligatoria (ESO) y Formación Profesional Básica (FPB): el $11.8 \%$ se encontraba cursando el $2^{\circ}$ año de la ESO, el $48.9 \%$ el $3^{\circ}$, el $36.6 \%$, el $4^{\circ}$ curso, y el $2.6 \%$ en $1^{\circ}$ y $2^{\circ}$ de FPB.

\section{Instrumentos}

Se utilizó un instrumento de autoinforme que incluía cuestiones referidas al sexo, edad, centro y curso de los y las participantes, y un instrumento referido a la competencia interpersonal en el marco del cortejo:

Cuestionario de Competencia Interpersonal en el Marco del Cortejo Adolescente (AICQc; Viejo et al., 2020). Se trata de una versión adaptada específicamente al contexto del cortejo adolescente del Interpersonal Competence Questionnaire (Buhrmester et al., 1988; Buhrmester, 1990), que sigue la misma estructura factorial que el original y queda compuesta por 35 ítems. Esta medida multidimensional se utilizó para medir el nivel de competencia sentimental que tenían los chicos y chicas durante el proceso de cortejo y en el inicio de la relación utilizando diferentes situaciones cotidianas en las que los adolescentes se pueden encontrar. La competencia sentimental se midió en torno a cinco factores independientes: iniciar relaciones ( 7 ítems; p.ej. Te sientes capaz de hacer todo lo posible por empezar nuevas amistades; $\alpha=.80$ ), asertividad y capacidad para decir no ( 8 ítems; p.e. Te sientes capaz de decir "no" cuando alguien que te gusta te pide que hagas algo que no quieres hacer; $\alpha=.82$ ), autorrevelación ( 7 ítems; p.ej. Te sientes capaz de contarle algo intimo sobre ti a alguien que te interesa o estás conociendo; $\alpha=.78$ ), dar apoyo emocional (7 ítems; p.ej. Te sientes capaz de hacer que alguien que te gusta o con quien empiezas a salir sienta que entiendes sus problemas; $\alpha=.85$ ) y gestionar conflictos (6 ítems; p.ej. Te sientes capaz de resolver problemas con alguien que te gusta o con quien empiezas a salir de forma que las cosas vayan a mejor en vez de peor; $\alpha=.80$ ). Los ítems están medidos en una escala likert de 5 puntos $(1=$ me siento torpe, $2=$ no se me da muy bien, $3=$ se me da medio bien, $4=$ soy bastante bueno/a en esto y $5=$ soy muy bueno/a en esto).

\section{Procedimiento}

El estudio fue aprobado por el Comité de Bioética y Seguridad de la Universidad de Córdoba y se siguieron los principios de la Declaración de Helsinki para su realización. 
El muestreo se llevó a cabo de modo incidental por accesibilidad. Se hizo una oferta abierta a distintos centros de la provincia de Córdoba en coordinación con la Delegación de Educación Provincial, y se contactó con aquellos centros que mostraron su interés y disponibilidad para participar en el estudio. Se tuvo un primer contacto con representantes de cada uno de los centros participantes donde se explicaron las bases del estudio y los objetivos que se dibujaban con esta investigación. Posteriormente, se acudió a cada centro para tener reuniones con el profesorado, el alumnado y las familias participantes, donde fueron informados del objetivo y procedimiento del estudio y se obtuvo el consentimiento informado. Asimismo, se informó al alumnado del carácter voluntario y anónimo de su participación y de la posibilidad de abandono del estudio si así lo deseaban. Finalmente, se administró el autoinforme presencialmente en el horario de tutoría que cada curso y centro tenía establecido, de manera que se ajustó el horario para que todos los cursos de un mismo centro pudiesen cumplimentar el cuestionario en el mismo día. La duración fue de, aproximadamente, 30 minutos.

\section{Análisis de datos}

El nivel de competencia sentimental se evaluó a través de las puntuaciones medias mostradas por los y las participantes en los cinco dominios descritos. Se realizaron análisis descriptivos utilizando los ítems correspondientes a cada dominio de forma independiente, para observar sus valores porcentuales. Para comprobar si existían diferencias significativas por sexo y edad se realizaron análisis de comparación de medias, utilizando las pruebas $\mathrm{T}$ de Student y ANOVA respectivamente. Comprobando la homogeneidad de las varianzas, se realizó la prueba post hoc mediante el procedimiento HSD Tukey (Field, 2009). Para las pruebas mencionadas, los tamaños del efecto fueron medidos con los coeficientes $d$ de Cohen para $\mathrm{T}$ de Student (efecto bajo $=0.20$; efecto medio $=0.50$; efecto alto $=0.80$ ) y Eta Cuadrado para ANOVA (pequeño $=.01 ;$ mediano $=.06$; grande $=.14)($ Cohen, 1992; Sun, Pan y Wan, 2010). La codificación y el análisis de los datos se realizaron utilizando el software estadístico SPSS 20.0 (IBM, Armonk, NY, USA).

\section{Resultados}

\section{Análisis descriptivo del nivel de competencia sentimental}

Iniciar relaciones sentimentales. Para esta primera dimensión, referida a la competencia para el inicio de la relación sentimental con aquella persona que ha despertado su interés erótico-sentimental, los chicos y chicas participantes reportaron sentirse hábiles y seguros de sus capacidades $(M=3.43 ; D T=.81)$. Más del $25 \%$ indicaron que esta tarea se les daba medio bien y más del $32 \%$ se consideraban bastante buenos/as a la hora de iniciar una relación sentimental. Analizando los diferentes ítems, los resultados mostraron que las habilidades para mantener una conversación con alguien nuevo y dar una buena primera impresión obtuvieron las puntuaciones más altas, con un $83.2 \%$ y un $70.7 \%$, respectivamente, de adolescentes que se sentían buenos o muy buenos en esas habilidades. Sin embargo, un $40.6 \%$ de los y las participantes se sentía torpe o sentía que no se le daba muy bien pedirle a alguien por el cual se sentía atraído, hacer algo juntos; igualmente, un $39.1 \%$ se sentía así respecto a presentarse a sí mismo a alguien que le atraía, y un 29.8\% respecto a llamarlo para quedar o hacer algo juntos (ver Tabla 1). 
Tabla 1.- Capacidad para iniciar relaciones sentimentales

\begin{tabular}{|c|c|c|c|c|c|}
\hline & \multicolumn{5}{|c|}{ Frecuencia de respuesta $(\%)$} \\
\hline & $\begin{array}{l}\text { Me } \\
\text { siento } \\
\text { torpe }\end{array}$ & $\begin{array}{l}\text { No se } \\
\text { me da } \\
\text { muy } \\
\text { bien }\end{array}$ & $\begin{array}{l}\text { Se me da } \\
\text { medio } \\
\text { bien }\end{array}$ & $\begin{array}{l}\text { Soy } \\
\text { bastante } \\
\text { bueno/a en } \\
\text { esto }\end{array}$ & $\begin{array}{l}\text { Soy muy } \\
\text { bueno/a } \\
\text { en esto }\end{array}$ \\
\hline $\begin{array}{l}\text { Te sientes capaz de pedirle a alguien que } \\
\text { te gusta o te resulta atractivo hacer algo } \\
\text { juntos como ir a ver un partido o ir al cine }\end{array}$ & 14.9 & 25.7 & 32.8 & 17.8 & 8.9 \\
\hline $\begin{array}{l}\text { Te sientes capaz de hacer todo lo posible } \\
\text { por empezar nuevas amistades }\end{array}$ & 4.4 & 10.8 & 25.3 & 32.6 & 26.8 \\
\hline $\begin{array}{l}\text { Te sientes capaz de hablar y mantener } \\
\text { conversaciones con alguien nuevo a quien } \\
\text { te gustaría conocer }\end{array}$ & 6 & 8.3 & 20.5 & 53.1 & 30.1 \\
\hline $\begin{array}{l}\text { Te sientes capaz de presentarte tú mismo/a } \\
\text { a alguien que te gustaría conocer o con } \\
\text { quien te gustaría salir }\end{array}$ & 17 & 22.1 & 26.9 & 20.1 & 13.9 \\
\hline $\begin{array}{l}\text { Te sientes capaz de llamar por teléfono } \\
\text { a alguien que te gusta atrae o con quien } \\
\text { empiezas a salir para quedar para veros y } \\
\text { hacer algo }\end{array}$ & 14.3 & 15.5 & 25.7 & 19.1 & 25.3 \\
\hline $\begin{array}{l}\text { Te sientes capaz de dar una buena primera } \\
\text { impresión cuando conoces a alguien con } \\
\text { quien te gustaría comenzar una amistad o } \\
\text { empezar a salir }\end{array}$ & 5 & 5.6 & 18.7 & 36.7 & 34 \\
\hline $\begin{array}{l}\text { Te sientes capaz de ir a fiestas o } \\
\text { actividades con gente nueva para hacer } \\
\text { nuevos amigos o conocer a más gente }\end{array}$ & 8.5 & 9.5 & 19.5 & 25.7 & 36.8 \\
\hline
\end{tabular}

Nota: se destacan en negrita los ítems que alcanzan mayores y menores puntuaciones dentro de la dimensión.

Asertividad y capacidad para decir no. Los resultados relativos a la segunda dimensión reportaron una puntuación media de $M=3.76(D T=.76)$. En relación a la propia percepción de la competencia para decir no cuando uno cree que es lo que debe decir, y considerando todos sus componentes, mostraron que alrededor de un $28 \%$ de los y las participantes consideraban que esta tarea se les daba medio bien; más de un $32 \%$ que eran bastante buenos y alrededor de un $36 \%$ se percibían como muy buenos. Concretamente, destacaron los comportamientos referidos a defender los derechos propios cuando la otra persona es grosera o no es respetuosa (el $80.6 \%$ se percibían como buenos o muy buenos en esa tarea) y decir "no" cuando alguien te pide hacer algo que no quieres (el 75.5\% se percibían como buenos o muy buenos en esa tarea). Sin embargo, ser capaz de decirle a alguien por el que se siente 
atracción que está haciendo algo que te avergüenza acumulaba un $25.4 \%$ de percepción de torpeza o poca habilidad (ver Tabla 2).

Tabla 2.- Asertividad y capacidad para decir no

\begin{tabular}{|c|c|c|c|c|c|}
\hline & \multicolumn{5}{|c|}{ Frecuencia de respuesta $(\%)$} \\
\hline & $\begin{array}{l}\text { Me } \\
\text { siento } \\
\text { torpe }\end{array}$ & $\begin{array}{l}\text { No se } \\
\text { me da } \\
\text { muy } \\
\text { bien }\end{array}$ & $\begin{array}{l}\text { Se me da } \\
\text { medio bien }\end{array}$ & $\begin{array}{l}\text { Soy bastante } \\
\text { bueno/a en } \\
\text { esto }\end{array}$ & $\begin{array}{l}\text { Soy muy } \\
\text { bueno/a en } \\
\text { esto }\end{array}$ \\
\hline $\begin{array}{l}\text { Te sientes capaz de decirle a alguien } \\
\text { que te gusta o con quien empiezas a } \\
\text { salir que no te gusta la forma en que te } \\
\text { ha estado tratando }\end{array}$ & 6.6 & 11.8 & 20 & 33.5 & 28.1 \\
\hline $\begin{array}{l}\text { Te siente capaz de decir "no" cuando } \\
\text { alguien que te gusta te pide que hagas } \\
\text { algo que no quieres hacer }\end{array}$ & 2.9 & 7.2 & 14.4 & 29.1 & 46.4 \\
\hline $\begin{array}{l}\text { Te sientes capaz de rechazar una } \\
\text { petición que no crees que sea adecuada } \\
\text { cuando la hace alguien que te gusta o } \\
\text { con quien empiezas a salir }\end{array}$ & 4.9 & 12.1 & 25.9 & 30.2 & 26.9 \\
\hline $\begin{array}{l}\text { Te sientes capaz de defender tus } \\
\text { derechos cuando alguien que te gusta o } \\
\text { con quien empiezas a salir es grosero o } \\
\text { no respeta }\end{array}$ & 1.6 & 6.2 & 11.6 & 28.9 & 51.7 \\
\hline $\begin{array}{l}\text { Te sientes capaz de decirle a alguien } \\
\text { que te gusta o con quien empiezas a } \\
\text { salir que está haciendo algo que te } \\
\text { avergüenza }\end{array}$ & 9.8 & 15.6 & 28.5 & 27 & 19.1 \\
\hline $\begin{array}{l}\text { Te sientes capaz de enfrentarte a } \\
\text { alguien que te gusta o con quien } \\
\text { empiezas a salir porque ha roto una } \\
\text { promesa }\end{array}$ & 5.2 & 13.2 & 25.4 & 31.8 & 24.3 \\
\hline $\begin{array}{l}\text { Te sientes capaz de decirle a alguien } \\
\text { que te gusta o con quien empiezas a } \\
\text { salir que ha hecho algo que ha herido } \\
\text { tus sentimientos }\end{array}$ & 4.4 & 11.4 & 20.2 & 33.1 & 30.8 \\
\hline $\begin{array}{l}\text { Te sientes capaz de decirle a alguien } \\
\text { que te gusta o con quien empiezas a } \\
\text { salir que ha hecho algo que te ha hecho } \\
\text { enfadar }\end{array}$ & 3.3 & 9 & 18.8 & 35 & 33.8 \\
\hline
\end{tabular}

Nota: se destacan en negrita los ítems que alcanzan mayores y menores puntuaciones dentro de la dimensión. 
Autorrevelación. La competencia para hablar espontáneamente de cosas propias con la persona que te gusta resultó con puntuaciones bajas $(M=3.37 ; D T=.77)$ en algunos de los comportamientos por los que se indagaba: en torno a un $26 \%$ se consideraba que la tarea no se le daba muy bien; más del $28 \%$ de los participantes consideraban que se les daba medio bien compartir información personal con la otra persona y más del $29 \%$ se consideraban bastante buenos en esto. El análisis de los comportamientos específicos por los que se indagaba señaló que la habilidad para contar algo íntimo o la habilidad para contar algo que te avergüenza sobre ti mismo a alguien que estás conociendo generaba las mayores inseguridades entre los chicos y chicas, con puntuaciones que alcanzaban el $32.1 \%$ y el $47.4 \%$ respectivamente, en la percepción de torpeza y poca habilidad. Comportamientos como abrirse a alguien que se empieza a conocer o compartir las ideas y sentimientos propios con esa persona alcanzaron un porcentaje de $66.6 \%$ y $69.8 \%$ en la percepción de considerarse bueno o muy bueno (ver Tabla 3).

Tabla 3.- Autorrevelación

\begin{tabular}{|c|c|c|c|c|c|}
\hline & \multicolumn{5}{|c|}{ Frecuencia de respuesta $(\%)$} \\
\hline & $\begin{array}{l}\text { Me } \\
\text { siento } \\
\text { torpe }\end{array}$ & $\begin{array}{l}\text { No se } \\
\text { me da } \\
\text { muy } \\
\text { bien }\end{array}$ & $\begin{array}{l}\mathrm{Se} \\
\text { me da } \\
\text { medio } \\
\text { bien }\end{array}$ & $\begin{array}{l}\text { Soy } \\
\text { bastante } \\
\text { bueno/a } \\
\text { en esto }\end{array}$ & $\begin{array}{l}\text { Soy } \\
\text { muy } \\
\text { bueno/a } \\
\text { en esto }\end{array}$ \\
\hline $\begin{array}{l}\text { Te sientes capaz de contarle algo intimo } \\
\text { sobre ti a alguien que te interesa o estás } \\
\text { conociendo }\end{array}$ & 16.5 & 25.6 & 28.5 & 17.8 & 11.6 \\
\hline $\begin{array}{l}\text { Te sientes capaz de dejar que alguien que } \\
\text { estás conociendo o con quien empiezas a } \\
\text { salir vea tu lado más sensible }\end{array}$ & 9.2 & 15.4 & 29.2 & 29.6 & 16.6 \\
\hline $\begin{array}{l}\text { Te sientes capaz de contarle algo de ti que } \\
\text { te avergüenza a alguien que te gusta o con } \\
\text { quien empiezas a salir }\end{array}$ & 21.3 & 26.1 & 24.6 & 18 & 10.1 \\
\hline $\begin{array}{l}\text { Te sientes capaz de abrirte a alguien que te } \\
\text { gusta o con quien empiezas a salir y dejar } \\
\text { que te conozca de verdad }\end{array}$ & 3.9 & 9.5 & 20 & 34.8 & 31.8 \\
\hline $\begin{array}{l}\text { Te sientes capaz de compartir con alguien } \\
\text { que te gusta o con quien empiezas a salir } \\
\text { tus ideas y sentimientos }\end{array}$ & 2.5 & 5.3 & 22.5 & 39.5 & 30.3 \\
\hline $\begin{array}{l}\text { Te sientes capaz de contarle a alguien que } \\
\text { te gusta o con quien empiezas a salir cosas } \\
\text { que no quieres que sepa todo el mundo }\end{array}$ & 12.2 & 17.4 & 30.3 & 23.6 & 16.6 \\
\hline
\end{tabular}

Nota: se destacan en negrita los items que alcanzan mayores y menores puntuaciones dentro de la dimensión. 
Apoyo emocional. La dimensión referida a dar apoyo afectivo fue la que, en términos generales, obtuvo las puntuaciones más altas $(M=4.21 ; D T=.64)$ : un $42 \%$ de los y las participantes se consideraba bastante bueno en esa tarea y más del $54 \%$ se consideraba muy bueno dando apoyo emocional a su incipiente pareja o persona elegida para el cortejo. En esta dimensión, todos los comportamientos obtenían puntuaciones similares, con altos porcentajes en la opción referida a sentirse buenos o muy buenos. La puntuación más alta se encontró en hacer que alguien que te atrae se sienta mejor cuando está triste o deprimido (54.8\% percibían ser muy buenos en esto), mientras que la más baja se refería a la habilidad para hacer que alguien que te atrae sienta que entiendes sus problemas (35.6\% puntuaban como muy buenos) (ver Tabla 4).

Tabla 4.- Dar apoyo emocional

\begin{tabular}{|c|c|c|c|c|c|}
\hline & \multicolumn{5}{|c|}{ Frecuencia de respuesta (\%) } \\
\hline & $\begin{array}{l}\text { Me } \\
\text { siento } \\
\text { torpe }\end{array}$ & $\begin{array}{l}\text { No se me } \\
\text { da muy } \\
\text { bien }\end{array}$ & $\begin{array}{l}\text { Se } \\
\text { me da } \\
\text { medio } \\
\text { bien }\end{array}$ & $\begin{array}{l}\text { Soy } \\
\text { bastante } \\
\text { bueno/a } \\
\text { en esto }\end{array}$ & $\begin{array}{l}\text { Soy muy } \\
\text { bueno/a } \\
\text { en esto }\end{array}$ \\
\hline $\begin{array}{l}\text { Te sientes capaz de ayudar a alguien que } \\
\text { te gusta a comprender sus pensamientos } \\
\text { y sentimientos para tomar una decisión } \\
\text { importante en su vida. Por ejemplo. elegir } \\
\text { qué quiere estudiar }\end{array}$ & 2.7 & 5.4 & 14.1 & 36.5 & 41.3 \\
\hline $\begin{array}{l}\text { Te sientas capaz de hacer que alguien que } \\
\text { te gusta o con quien empiezas a salir sienta } \\
\text { que entiendes sus problemas }\end{array}$ & 1.6 & 5.1 & 15.8 & 42 & 35.6 \\
\hline $\begin{array}{l}\text { Te sientes capaz de ayudar a alguien que } \\
\text { te gusta o con quien empiezas a salir a } \\
\text { comprender mejor sus problemas }\end{array}$ & .4 & 3.7 & 14.9 & 35.7 & 45.3 \\
\hline $\begin{array}{l}\text { Te sientes capaz de ayudar a alguien que } \\
\text { te gusta o con quien empiezas a salir a } \\
\text { enfrentarse a la presión o a momentos } \\
\text { difíciles }\end{array}$ & .6 & 3.1 & 14.2 & 40 & 42.1 \\
\hline $\begin{array}{l}\text { Te sientes capaz de hacer que alguien que } \\
\text { te gusta o con quien empiezas a salir se } \\
\text { sienta mejor cuando está triste o deprimido }\end{array}$ & 1 & 3.3 & 8.8 & 32.1 & 54.8 \\
\hline $\begin{array}{l}\text { Te sientes capaz de mostrar que realmente } \\
\text { te interesa o te importa cuando alguien que } \\
\text { te gusta o con quien empiezas a salir te } \\
\text { cuenta sus problemas }\end{array}$ & .6 & 2.5 & 13.5 & 35.4 & 48.1 \\
\hline $\begin{array}{l}\text { Te sientes capaz de dar consejos que } \\
\text { puedan ayudar a alguien que te gusta o con } \\
\text { quien empiezas a salir }\end{array}$ & 1.3 & 5 & 10.8 & 33.1 & 49.8 \\
\hline
\end{tabular}

Nota: se destacan en negrita los ítems que alcanzan mayores puntuaciones dentro de la dimensión. 
Gestionar conflictos en el cortejo. Por último, considerando las puntuaciones globales de esta dimensión $(M=3.83 ; D T=.71)$, un $32 \%$ de los y las participantes consideraban que se les daba medio bien superar los problemas con la persona que les gustaba; más de un $35 \%$ se consideraban bastante buenos solucionando un conflicto y más de un $37 \%$ se consideraban muy buenos. El análisis pormenorizado de los comportamientos permitió identificar

Tabla 5.- Gestionar conflictos

\begin{tabular}{|c|c|c|c|c|c|}
\hline & \multicolumn{5}{|c|}{ Frecuencia de respuesta (\%) } \\
\hline & $\begin{array}{l}\mathrm{Me} \\
\text { siento } \\
\text { torpe }\end{array}$ & $\begin{array}{l}\text { No se } \\
\text { me da } \\
\text { muy } \\
\text { bien }\end{array}$ & $\begin{array}{l}\text { Se } \\
\text { me da } \\
\text { medio } \\
\text { bien }\end{array}$ & $\begin{array}{l}\text { Soy } \\
\text { bastante } \\
\text { bueno/a } \\
\text { en esto }\end{array}$ & $\begin{array}{l}\text { Soy } \\
\text { muy } \\
\text { bueno/a } \\
\text { en esto }\end{array}$ \\
\hline $\begin{array}{l}\text { Te sientes capaz de resolver } \\
\text { problemas con alguien que te gusta o } \\
\text { con quien empiezas a salir de forma } \\
\text { que las cosas vayan a mejor en vez } \\
\text { de peor }\end{array}$ & 1.4 & 6.2 & 19.5 & 35.3 & 37.6 \\
\hline $\begin{array}{l}\text { Te sientes capaz de gestionar los } \\
\text { problemas con alguien que te gusta o } \\
\text { con quien empiezas a salir de forma } \\
\text { que, a la larga, os haga felices a } \\
\text { ambos }\end{array}$ & 1.4 & 5.2 & 17.8 & 39.7 & 35.9 \\
\hline $\begin{array}{l}\text { Te sientes capaz de resolver los } \\
\text { problemas con alguien que te gusta o } \\
\text { con quien empiezas a salir de forma } \\
\text { que ninguno de los dos salga herido } \\
\text { o resentido }\end{array}$ & 3.1 & 5.6 & 20.3 & 40 & 31.1 \\
\hline $\begin{array}{l}\text { Te sientes capaz de tratar los } \\
\text { problemas con alguien que te gusta o } \\
\text { con quien empiezas a salir de forma } \\
\text { que no siempre salga perdiendo la } \\
\text { misma persona }\end{array}$ & 2.5 & 10.1 & 26.7 & 38.1 & 22.6 \\
\hline $\begin{array}{l}\text { Te sientes capaz de resolver los } \\
\text { conflictos con alguien que te gusta o } \\
\text { con quien empiezas a salir antes de } \\
\text { que se conviertan en peleas }\end{array}$ & 1.7 & 7.5 & 22.8 & 35.7 & 32.2 \\
\hline $\begin{array}{l}\text { Te sientes capaz de superar } \\
\text { rápidamente los problemas con } \\
\text { alguien que te gusta o con quien } \\
\text { empiezas a salir }\end{array}$ & 4.2 & 13.5 & 32.9 & 32.4 & 17 \\
\hline
\end{tabular}

Nota: se destacan en negrita los ítems que alcanzan mayores y menores puntuaciones dentro de la dimensión. 
que un alto porcentaje de los chicos y chicas se sentían buenos o muy buenos gestionando problemas de forma que a la larga los hiciera sentir felices (75.6\%), resolviendo problemas para evitar que fueran a peor $(72.9 \%)$ y tratando que ninguna de las partes saliera herida o resentida $(71.1 \%)$. Sin embargo, comportamientos como gestionar los problemas para que no salga siempre perdiendo la misma persona (12.6\%) o superar rápidamente los problemas que surgen con alguien que te atrae (17.7\%) obtuvo los porcentajes más altos en cuanto a percepción personal de torpeza o dificultad (ver Tabla 5).

En general, y atendiendo a las puntuaciones medias obtenidas en cada una de las cinco dimensiones con las que se ha medido el constructo competencia social, los resultados mostraron un nivel medio en la percepción de competencia sentimental, situándose por orden creciente autorrevelación $(M=3.37 ; D T=.77)$, iniciar relaciones $(M=3.43 ; D T=.81)$, asertividad y capacidad para decir no $(M=3.75 ; D T=.76)$, gestionar conflictos $(M=3.83$; $D T=.71)$, y dar apoyo emocional $(M=4.21 ; D T=.65)$.

\section{Diferencias por sexo y edad en los dominios de la competencia sentimental}

Para ahondar en la comprensión de este constructo a lo largo de los años adolescentes, se analizaron las posibles diferencias que pudieran establecerse entre el sexo y la edad para los cinco dominios establecidos.

En cuanto al sexo, los resultados solo mostraron diferencias significativas en la dimensión de asertividad y capacidad para decir no $(t(520)=-1405 ; p=0.047 ; \mathrm{d}=0.13)$. Aunque con un tamaño del efecto bajo, las chicas $(M=27.04 ; D T=5.58)$ mostraron ser más asertivas y más capaces de decir que no a la persona que les gusta cuando esta hace algo que no les parece bien, que los chicos $(M=26.36 ; D T=4.95)$.

Respecto a la edad, se encontraron diferencias significativas en cuatro de las dimensiones de la competencia sentimental: iniciar relaciones $\left(F(6,520)=3.80 ; p=0.01 ; \eta^{2}=0.043\right)$; asertividad y capacidad para decir no $\left(F(6,520)=2.29 ; p=0.03 ; \eta^{2}=0.027\right)$; dar apoyo emocional $\left(F(6,520)=2.29 ; p=0.03 ; \eta^{2}=0.026\right)$; y gestionar conflictos $(F(6,520)=2.88$; $\left.p=0.00 ; \eta^{2}=0.033\right)$. Las pruebas post hoc mostraron que las diferencias se localizaban entre los y las adolescentes de 13 y 14 años versus los y las adolescentes de 16 años o más, siendo los y las de este último rango de edad quienes obtuvieron las puntuaciones más altas (ver Tabla 6).

\section{Discusión y Conclusiones}

El objetivo de este trabajo era avanzar en el análisis de las competencias sentimentales específicas que los chicos y chicas participantes tenían en el momento de abordar esta tarea psicoevolutiva. Los resultados han apuntado que, en términos generales, chicos y chicas afrontan la tarea con cierta inexperiencia que se refleja, sobre todo, en determinadas dimensiones. Si bien parecen tener más o mejores herramientas para la gestión del apoyo emocional que implican estas relaciones, se encuentran con más dificultades cuando tienen que abordar conductual y actitudinalmente una relación que se presenta como una novedad que los involucra en su identidad y en su proyecto de búsqueda de la intimidad y del apoyo mutuo en una relación que, aunque muy deseada, no siempre se ajusta a las expectativas y 
Tabla 6.- Diferencias significativas por edad en los dominios de la Competencia Sentimental

\begin{tabular}{|c|c|c|c|c|c|c|c|}
\hline Edad (n) & M & $D T$ & $F$ & $p$ & $g l$ & $\eta^{2}$ & Diferencia de medias \\
\hline \multicolumn{8}{|c|}{ Iniciar Relaciones } \\
\hline $13(37)$ & 3.29 & .68 & \multirow{7}{*}{3.810} & \multirow{7}{*}{.001} & \multirow{7}{*}{6,506} & \multirow{7}{*}{0.043} & \\
\hline $14(164)$ & 3.28 & .87 & & & & & \\
\hline 15 (197) & 3.45 & .80 & & & & & $13-18$ años: $0.865^{*}$ \\
\hline $16(76)$ & 3.53 & .72 & & & & & $14-17$ años: $0.540^{*}$ \\
\hline $\begin{array}{l}17(27) \\
18(19)\end{array}$ & 3.82 & .72 & & & & & $14-18$ años: $0.873^{*}$ \\
\hline \multirow[t]{2}{*}{$19(2)$} & 4.15 & .57 & & & & & \\
\hline & 3.5 & .50 & & & & & \\
\hline \multicolumn{8}{|c|}{ Asertividad y capacidad para decir no } \\
\hline $13(37)$ & 3.37 & .82 & & & & & \multirow{7}{*}{$\begin{array}{l}13-14 \text { años: } 0.425^{*} \\
13-16 \text { años: } 0.528^{*}\end{array}$} \\
\hline $14(164)$ & 3.79 & .75 & & & & & \\
\hline $15(197)$ & 3.73 & .77 & & & & & \\
\hline $16(76)$ & 3.90 & .67 & 2.296 & .034 & 6,506 & 0.027 & \\
\hline $\begin{array}{l}17(27) \\
18(19)\end{array}$ & 3.85 & .66 & & & & & \\
\hline \multirow[t]{2}{*}{$19(2)$} & 3.68 & .89 & & & & & \\
\hline & 3.50 & .70 & & & & & \\
\hline \multicolumn{8}{|c|}{ Autorrevelación } \\
\hline $13(37)$ & 3.34 & .68 & \multirow{7}{*}{2.100} & \multirow{7}{*}{.052} & \multirow{7}{*}{6,506} & \multirow{7}{*}{0.024} & \multirow{7}{*}{$14-17$ años: $0.528^{*}$} \\
\hline $14(164)$ & 3.24 & .87 & & & & & \\
\hline 15 (197) & 3.40 & .80 & & & & & \\
\hline $16(76)$ & 3.40 & .72 & & & & & \\
\hline $\begin{array}{l}17(27) \\
18(19)\end{array}$ & 3.76 & .72 & & & & & \\
\hline \multirow[t]{2}{*}{$19(2)$} & 3.48 & .57 & & & & & \\
\hline & 3.50 & .50 & & & & & \\
\hline \multicolumn{8}{|c|}{ Dar apoyo emocional } \\
\hline $13(37)$ & 4.14 & .70 & \multirow{7}{*}{2.290} & \multirow{7}{*}{.034} & \multirow{7}{*}{4,512} & \multirow{7}{*}{0.026} & \multirow{7}{*}{$\begin{array}{l}\text { No hubo diferencias } \\
\text { post-hoc }\end{array}$} \\
\hline $14(164)$ & 4.11 & .68 & & & & & \\
\hline \multirow{5}{*}{$\begin{array}{l}15(197) \\
16(76) \\
17(27) \\
18(19) \\
19(2)\end{array}$} & 4.21 & .63 & & & & & \\
\hline & 4.27 & .57 & & & & & \\
\hline & 4.42 & .58 & & & & & \\
\hline & 4.61 & .36 & & & & & \\
\hline & 4.92 & .10 & & & & & \\
\hline \multicolumn{8}{|c|}{ Gestionar conflictos } \\
\hline $13(37)$ & 3.50 & .68 & \multirow{7}{*}{2.882} & & & & \\
\hline $14(164)$ & 3.76 & .87 & & & & & \\
\hline 15 (197) & 3.88 & .80 & & & & & \\
\hline $16(76)$ & 3.86 & .72 & & .009 & 4,512 & 0.009 & 13-17 años: $0.678^{*}$ \\
\hline $\begin{array}{l}17(27) \\
18(19)\end{array}$ & 4.18 & .72 & & & & & \\
\hline $19(2)$ & 3.73 & .57 & & & & & \\
\hline & 4.08 & .50 & & & & & \\
\hline
\end{tabular}


a las competencias que se autoperciben como propias. Se trata de comportamientos que requieren poner en práctica habilidades de naturaleza diversa: arriesgar invitaciones a realizar actividades conjuntas sin saber cómo se interpreta dicha iniciativa; invitar a procesos íntimos a alguien al que se conoce relativamente poco; expresar espontáneamente cosas hasta el momento quizás pertenecientes al núcleo de la intimidad personal, compartiendo ideas y sentimientos que no se comparten en escenarios sociales de grupo; gestionar conflictos que pueden tener un contenido muy novedoso sin que ello signifique una ruptura que a veces no se desea o que aun deseándola suele tener en cuenta los sentimientos del otro protagonista; entre otros matices de la complejidad sentimental, actitudinal y conductual de este tipo de procesos.

Los resultados del estudio realizado muestran que chicos y chicas afrontan sus relaciones con niveles de competencia similares, sin embargo, ellas se sienten más capaces de ser asertivas y decir no, lo que podría interpretarse como el resultado de las últimas campañas sociales como la del \#MeToo, que se han dirigido de forma específica a este aspecto (ClarkParsons, 2021). Pese que la adolescencia parece ser un periodo más o menos estable para este desarrollo competencial, sí pueden identificarse dos grandes momentos que establecen diferencias significativas: la adolescencia temprana (12-13 años) y la adolescencia tardía (16/17 años en adelante). Las diferencias significativas en las distintas dimensiones han aparecido, lógicamente, apuntando que la experiencia de los y las adolescentes de mayor edad tienen mejores niveles de competencia para afrontar estas situaciones. Si bien el carácter transversal de estudio no deja de ser una limitación para comprobar la verdadera evolución de estas competencias, este resultado no hace sino subrayar la necesidad de contribuir a la formación de los y las jóvenes en la adquisición de competencias relacionales en el contexto de la pareja, sin dejar que el proceso resulte un aprendizaje por ensayo-error. Futuros trabajos longitudinales que aborden este tema podrían beneficiarse de estas prácticas y comprobar el cambio efectivo de una formación específica.

Siendo aun una línea de trabajo incipiente, los resultados de este estudio subrayan la necesidad de apoyar a los y las jóvenes en su proceso de maduración sentimental y de desarrollo competencial para la formación de relaciones sentimentales ajustadas y de calidad que, a su vez, eviten factores de riesgo como la violencia. No obstante, una de las limitaciones que presenta este trabajo es precisamente no contar con medidas objetivas de la calidad de las relaciones que permitan establecer esta relación de forma empírica, objetivo que deberá abordarse en futuros estudios.

Pese a esta evidencia. desde la perspectiva psicoeducativa, la mayoría de las intervenciones que se han desarrollado en las tres últimas décadas han sido con la intención de reducir, eliminar o evitar la aparición de conductas agresivas en las relaciones sentimentales adolescentes (De La Rue et al., 2017; Jennings et al., 2017), dejando de lado la promoción positiva de estas relaciones. Asociada a buenos índices de bienestar, de satisfacción con la relación y de una mejor toma de decisiones entre los y las adolescentes, el trabajo para el desarrollo de la competencia sentimental surge como elemento clave y necesario (Davila et al., 2017) no solo para el crecimiento personal y desarrollo de relaciones saludables, sino también para la prevención de comportamientos de riesgo en el seno de las primeras relaciones eróticosentimentales. 
Para contribuir en esta línea, y en base a los resultados obtenidos, se hace una propuesta de intervención que contempla ambas dimensiones: el desarrollo positivo de las dimensiones de la competencia sentimental y la necesaria prevención de la violencia. El modelo NoViCo (No Violencia en el Cortejo; Viejo y Ortega-Ruiz, 2020) se estructura en torno a seis sesiones dirigidas respectivamente a (1) la competencia para iniciar relaciones; (2) la competencia de autorrevelación; (3) la capacidad para ser asertivos/as en el proceso de cortejo e inicio de una relación sentimental; (4) el análisis de los conflictos que pueden tener lugar durante el proceso de cortejo e inicio de las relaciones sentimentales; (5) la identificación de situaciones de violencia dentro de la pareja y el desarrollo de habilidades para prevenirla; y (6) el reconocimiento del riesgo que supone mantener una relación de pareja disfuncional y el desarrollo de habilidades para la ruptura. Estas dimensiones tienen una correspondencia directa con las dimensiones consideradas parte del desarrollo de la Competencia Interpersonal en el marco del cortejo. Se trata, por tanto, de una propuesta de trabajo que incide en la necesidad de formación de los chicos y chicas en sus habilidades para la gestión efectiva de las relaciones sentimentales (Davila et al., 2021). El fin último de este programa es fomentar un cambio actitudinal, emocional y comportamental en el alumnado, a través de una metodología activa y participativa que otorgue el protagonismo a los y las adolescentes y preste especial atención a su narrativa, así como que contemple la realización de dinámicas grupales y la utilización del juego como recurso de aprendizaje.

Este estudio representa un primer avance sobre lo que debe ser un estudio más complejo y completo, tomando en cuenta datos longitudinales que permitan valorar plenamente el desarrollo de la Competencia Sentimental a lo largo de la etapa adolescente, así como implementar y evaluar la propuesta de intervención de forma que se valoren el posible beneficio que aportaría a estas dimensiones competenciales.

\section{Agradecimientos al siguiente proyecto:}

Proceso Psicoevolutivo y Educativo del Cortejo Adolescente: Competencia Sentimental y Ajuste Social (UCO-1380774), financiado en el marco del Programa Operativo FEDER Andalucía 2014-2020.

\section{Referencias}

Blakemore, S. J. (2008). The social brain in adolescence. Nature Reviews Neuroscience, 9(4), 267-277. https://doi. org/10.1038/nrn2353

Bouchey, H. A. (2007). Perceived romantic competence, importance of romantic domains, and psychosocial adjustment. Journal of Clinical Child and Adolescent Psychology, 36(4), 503-514. https://doi. org/10.1080/15374410701653120

Buhrmester, D. (1990). Intimacy of friendship, interpersonal competence, and adjustment during preadolescence and adolescence. Child Development, 61(4), 1101-1111. https://doi.org/10.1111/j.1467-8624.1990.tb02844.x

Buhrmester, D., Furman, W., Wittenberg, M. T., y Reis, H. T. (1988). Five domains of interpersonal competence in peer relationships. Journal of Personality and Social Psychology, 55(6), 991-1008. https://doi.org/10.1037/00223514.55 .6 .991 
Clark-Parsons, R. (2021). "I see you, I believe you, I stand with you":\# MeToo and the performance of networked feminist visibility. Feminist Media Studies, 27(3), 362-380.

Cohen, J. (1992). A power primer. Psychological bulletin, 112(1), 155-159. https://doi.org/10.1037//0033-2909.112.1.155

Connolly, J., Furman, W., y Konarski, R. (2000). The role of peers in the emergence of heterosexual romantic relationships in adolescence. Child Development, 71(5), 1395-1408. https://doi.org/10.1117/1467-8624.00235

Davila, J., Mattanah, J., Bhatia, V., Latack, J. A., Feinstein, B. A., Eaton, N. R., Daks, J. S., Kumar, S. A., Lomash, E. F., Mccormick, M., y Zhou, J. (2017). Romantic competence, healthy relationship functioning, and well-being in emerging adults. Personal Relationships, 24(1), 162-184. https://doi.org/10.1117/pere.12175

Davila, J., Steinberg, S. J., Miller, M. R., Stroud, C. B., Starr, L. R., y Yoneda, A. (2009). Assessing romantic competence in adolescence: The Romantic Competence Interview. Journal of Adolescence, 32(1), 55-75. https://doi. org/10.1016/j.adolescence.2007.12.001

Davila, J., Zhou, J., Norona, J., Bhatia, V., Mize, L., y Lashman, K. (2021). Teaching romantic competence skills to emerging adults: A relationship education workshop. Personal Relationships, 28(2), $251-275$.

De La Rue, L., Polanin, J. R., Espelage, D. L., y Pigott, T. D. (2017). A meta-analysis of school-based interventions aimed to prevent or reduce violence in teen dating relationships. Review of Educational Research, 87(1), 7-34. https://doi.org/10.3102/0034654316632061

Delgado, I., Oliva, A., y Sánchez-Queija, I. (2011). Apego a los iguales durante adolescencia y la adultez emergente. Anales de Psicología / Annals of Psychology, 27(1), 155-163.

Dunphy, D. C. (1963). The social structure of urban adolescent peer groups. Sociometry, 26(2), 230-246. https://doi. org/10.2307/2785909

Field, A. (2009). Discovering statistics using SPSS. SAGE. https://doi.org/10.1234/12345678

Finkel, E. J., Simpson, J. A., y Eastwick, P. W. (2017). The psychology of close relationships: Fourteen core principles, Annual Review of Psychology, 68(1), 383-411. https://doi.org/10.1146/annurev-psych-010416-044038

Foshee, V. A., Reyes, H. L. M., Ennett, S. T., Suchindran, C., Mathias, J. P., Karriker-Jaffe, K. J., Bauman, K. E., y Benefield, T. S. (2011). Risk and protective factors distinguishing profiles of adolescent peer and dating violence perpetration. The Journal of Adolescent Health,48(4), 344-350. https://doi.org/10.1016/j. jadohealth.2010.07.030

Gómez-López, M., Viejo, C., y Ortega-Ruiz, R. (2019). Well-being and romantic relationships: A systematic review in adolescence and emerging adulthood. International Journal of Environmental Research and Public Health, 16(13), 2415. https://doi.org/10.3390/ijerph16132415

Gorrese, A., y Ruggieri, R. (2012). Peer attachment: A meta-analytic review of gender and age differences and associations with parent attachment. Journal of Youth and Adolescence, 47(5), 650-672. https://doi. org/10.1007/s10964-012-9759-6

Haselschwerdt, M. L., Savasuk-Luxton, R., y Hlavaty, K. (2017). A methodological review and critique of the "intergenerational transmission of violence" literature. Trauma, Violence, \& Abuse, 20(2), 168-182. https://doi. org/10.1177/1524838017692385

Jennings, W. C., Okeem, C., Piquero, A. R., Sellers, C. S., Theobald, D., y Farrington, D. P. (2017). Dating and intimate partner violence among young persons ages 15-30: Evidence from a systematic review. Aggression and Violent Behavior, 33, 107-125. https://doi.org/10.1016/j.avb.2017.01.007

Moksnes, U. K., y Haugan, G. (2015). Stressor experience negatively affects life satisfaction in adolescents: the positive role of sense of coherence. Quality of Life Research, 24(10), 2473-2481. https://doi.org/10.1007/s11136015-0977-8

Ortega-Ruiz, R., y Sánchez, V. (2011). Juvenile dating and violence. In C. Monks y I. Coyne (Eds.), Bullying en different context (pp. 1-39). Cambridge University Press.

Pellegrini, A. (2010). The role of physical activity in the development and function of human juveniles' sex segregation. Behaviour, 147(13), 1633-1656. https://doi.org/10.1163/000579510X535262 
Pellegrini, A. D. (2003). Perceptions and functions of play and real fighting in early adolescence. Child Development, 74(5), 1522-1533. https://doi.org/10.1111/1467-8624.00620

Pringle, J., Mills, K. L., McAteer, J., Jepson, R., Hogg, E., Anand, N., y Blakemore, S. J. (2017). The physiology of adolescent sexual behaviour: A systematic review. Cogent Social Sciences, 3(1), 1-14. https://doi.org/10.1080/23 311886.2017 .1368858

Romera, E.-M. M., Cano, J.-J. J., García-Fernández, C.-M. M., y Ortega-Ruiz, R. (2016). Cyberbullying: Social competence, motivation and peer relationships. Comunicar, 24(48), 71-79. https://doi.org/10.3916/C48-2016-07

Savickaite, R., Dijkstra, J. K., Kreager, D., Ivanova, K., y Veenstra, R. (2020). Friendships, perceived popularity, and adolescent romantic relationship debut. The Journal of Early Adolescence, 40(3), 377-399. https://doi. org/10.1177/0272431619847530

Shulman, S., Davila, J., y Shachar-Shapira, L. (2011). Assessing romantic competence among older adolescents. Journal of Adolescence, 34(3), 397-406. https://doi.org/10.1016/j.adolescence.2010.08.002

Stroud, C. B., y Davila, J. (2008). Pubertal timing and depressive symptoms in early adolescents: The roles of romantic competence and romantic experiences. Journal of Youth and Adolescence, 37(8), 953-966. https://doi.org/10.1007/s10964-008-9292-9

Sun, S., Pan, W., y Wang, L. L. (2010). A comprehensive review of effect size reporting and interpreting practices in academic journals in education and psychology. Journal of Educational Psychology, 102(4), 989-1004.

Viejo, C. (2014). Physical dating violence: Towards a comprehensible view of the phenomenon. Infancia y Aprendizaje, 37(4), 785-815. https://doi.org/10.1080/02103702.2014.977110

Viejo, C., Toledano, N., y Ortega-Ruiz, R. (2020). Romantic competence and adolescent courtship: The multidimensional nature of the construct and differences by age and gender. International Journal of Environmental Research and Public Health, 17(14), 5223. https://doi.org/10.3390/ijerph17145223

Viejo, C. y Ortega-Ruiz, R. (2020). Programa NoViCo, No Violencia en el Cortejo. Un programa de Prevención de la Violencia en el Cortejo e inicio de las Relaciones de Pareja en Educación Secundaria. (Documento no publicado)

Zhou, J., Bhatia, V., Luginbuehl, T., \& Davila, J. (2021). The association between romantic competence and couple support behaviors in emerging adult couples. Journal of Social and Personal Relationships, 38(3), 10151034. 